\title{
ESTUDIO DEL GÉNERO BATRACHOSPERMUM ROTH.: B. BORYANUM SIROD. Y B. MONILIFORME ROTH. EN LA PROVINCIA DE CASTELLÓN, ESPAÑA.
}

\author{
Mercedes PREFASI y Marina ABOAL
}

RESUMEN. Estudio del género Batrachospermum Roth.: B. boryanum Sirod. y B. moniliforme Roth. en la provincia de Castellón, España. En una prospección extensiva realizada en 175 fuentes de la provincia de Castellón, se ha recolectado Batrachospermum en un total de 13 localidades. Las especies identificadas son B. moniliforme (Roth.) y B. boryanum (Sirod.), (que no había sido citada con anterioridad para España). Se presentan las principales características físico-químicas de las aguas en las que se desarrollan.

Palabras clave. Batrachospermum, Rodofíceas, fuentes, Castellón, España.

ABSTRACT. Study of the genus Batrachospermum Roth.: B. boryanum Sirod. and B. moniliforme Roth. in Castellón province, Spain. In the prospection of 175 springs of the Castellón province, Batrachospermum has been collected in 13 stations. Only two species have been identified: $B$. moniliforme (Roth.) and $B$. boryanum (Sirod.),(a new record for Spain). The main physico-chemical parameters of water for each species are presented.

Key Words. Batrachospermum, Rodophyta, spring, Castellón, Spain.

\section{INTRODUCCIÓN}

La provincia de Castellón de la Plana, situada en el Levante de la Península Ibérica, está comprendida entre la Cordillera Ibérica y la Cordillera Costero-Catalana. Los materiales geológicos predominantes son carbonatados, principalmente calizas, margas y dolomías del Cretácico en el oeste, y arcillas, margas, dolomías y areniscas del Triásico en el suroeste y centro-sur (I.G.M.E., 1985). Es destacable el gran número de fuentes existentes en esta zona geográfica.
Un primer paso en el estudio de la flora ficológica de la provincia es el presente trabajo, que se centra en los rodófitos (Rhodophyceae), y más concretamente en las Batracospermáceas de las fuentes (fig 1).

Hasta el momento, en España se han citado cinco especies de Batrachospermum (Alvarez Cobelas, 1984). En la vertiente mediterránea se han citado cuatro especies para Cataluña (Cambra, 1985). Los estudios realizados más recientemente se limitan a aumentar su distribución a algunas localidades del NE y SE peninsular (Aboal, 1989; Sabater et al., 1989). 


\section{MATERIALES Y MÉTODOS}

Las muestras fueron recolectadas de forma manual y conservadas tras su recolección en una solución de formaldehido al $4 \%$. Alternativamente se realizaron pliegos de herbario con material prensado que se conservan en el Herbario MUB de la Universidad de Murcia.

Las monografías utilizadas para la identificación taxonómica fueron: Bourrelly (1970); Israelson (1942); Povoa Dos Reis (1974) y Starmach (1977).

\section{RESULTADOS Y DISCUSIÓN}

De las 175 fuentes estudiadas, solo se ha identificado Batrachospermum en un total de 13 (tab. I), en diez ocasiones Batrachospermum moniliforme Roth. (1, 2, 3, 4, 5, 7, 8, 1 0, 11, 13) y en otras tres Batrachospermun boryanum Sirodot $(6,9,12)$, que se cita por primera vez para España. La mayoría de estas fuentes se localizan en la zona oeste y centro-sur de la provincia.

Los talos cladomianos de $B$. boryanum pueden alcanzar un tamaño de $5 \mathrm{~cm}$ y presen-

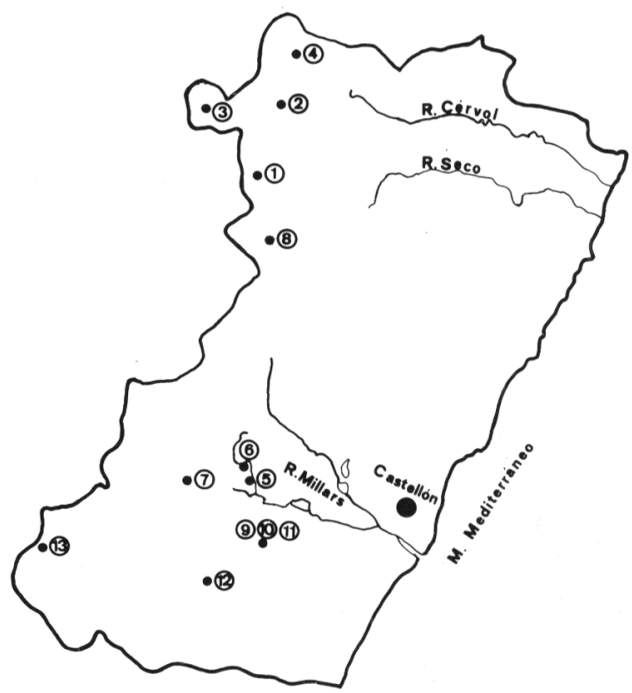

Figura 1. Localización de las fuentes con Batrachospermum en la provincia de Castellón. Geographical localization of the sources with Batrachospermum in the Castellón province.

\begin{tabular}{lllcrc}
\hline $\mathrm{N}^{\circ}$ & Localidad & Municipio & UTM & $\begin{array}{c}\text { Altitud } \\
\text { msnm }\end{array}$ & Fecha \\
\hline 1 & Ineso & Portell & 30TYK3190 & 1070 & $18 / 08 / 89$ \\
2 & Panera & Forcall & 30TYL3703 & 680 & $21 / 08 / 89$ \\
3 & Canaleta & Olocau & 30TYL2501 & 990 & $23 / 08 / 89$ \\
4 & Beana & Sorita & 30TYL3911 & 665 & $16 / 09 / 90$ \\
5 & StaCruz & Argelita & 30TYK2536 & 290 & $23 / 09 / 89$ \\
6 & Valentina & Ludiente & 30TYK2342 & 440 & $09 / 11 / 89$ \\
7 & Señor & Arañuel & 30TYK1240 & 480 & $07 / 04 / 90$ \\
8 & Gracieta & Villafranca & 30TYK3582 & 1240 & $09 / 03 / 90$ \\
9 & Riu & Sueras & 30SYK2726 & 300 & $29 / 04 / 90$ \\
10 & Monte Castro & Sueras & 30SYK2424 & 420 & $29 / 04 / 90$ \\
11 & Balseta & Sueras & 30SYK2827 & 400 & $29 / 04 / 90$ \\
12 & Donace & Algimia & 30SYK1922 & 520 & $29 / 06 / 90$ \\
& & Almonacid & & & \\
13 & Tejería & ElToro & 30SYK9127 & 1020 & $16 / 07 / 90$
\end{tabular}

Tabla 1. Lista de localidades con indicación de coordenadas UTM, altitud y fecha de prospección. List of localities with indication of UTM coordenates, altitude and prospection date. 

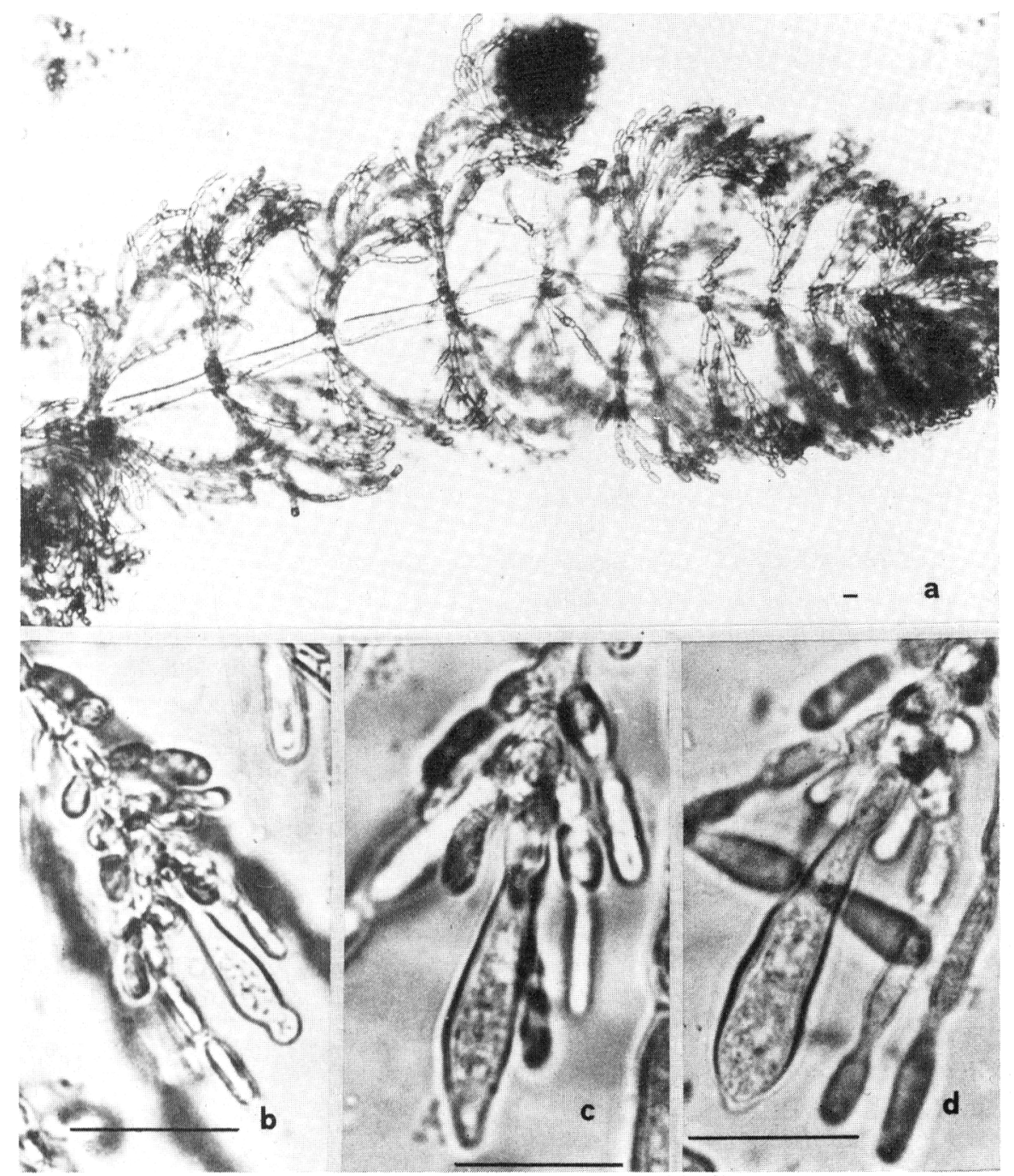

Figura 2. a: Aspecto general con pleuridios recurvados. b-d: Variabilidad de las tricóginas de $B$. boryanu, (La escala representa $10 \mathrm{~mm}$ ). a: General view of curved pleurides. $b$-d: Variability of the tricogines of $B$. boryanum. 
tan una coloración rosado-violácea. Los carposporófitos (50-144 $\mu \mathrm{m}$ de diámetro) se desarrollan en número reducido (1-3), en las porciones externas de los verticilos.

El aspecto coraliforme (fig. 2, a) que presenta esta especie macroscópicamente se debe a la escasa longitud de las zonas internodales (88-800 $\mu \mathrm{m}$ ) (fig. 2, b) y a la curvatura más o menos acusada de los pleuridios y de los ápices de los filamentos (fig. 2, c; fig. 3, d). Este carácter morfológico junto con la escasez de pelos en las ramas (fig. 2, d) permite separar con relativa facilidad este taxón de otros afines (Sheath \& Burkholder, 1983). Sin embargo, la validez del carácter de los pelos es dudosa ya que está comprobado que su densidad y longitud dependen del contenido en nutrientes del medio (Gibson \& Whitton, 1987).

Las tricóginas (26.4-38.4 $\mu \mathrm{m})$, sésiles, presentan una forma muy variable; pasan de lageniformes a elipsoides en el mismo individuo, con todo tipo de transiciones (fig. 2, e-g; fig. 3, a-c). Este hecho ya fue puesto en evidencia por Sheath \& Burkholder (1983), que propusieron el cambio de la especie a la sección Moniliformia. Tradicionalmente Bourrelly (1970); Israelson (1942); Povoa Dos Reis (1974) y Starmach (1977) consideraban la tricógina elipsoide y la incluían en la sección Helminthoidea.

En la mayoría de las poblaciones estudiadas en la provincia de Castellón se observó un predominio de especímenes dioicos, aunque también con cierta frecuencia se recolectaron monoicos. En Estados Unidos parece predominar la dioecia (Sheath \& Burkholder, 1983), mientras que en Suecia (Israelson, 1942), Portugal (Povoa Dos Reis, 1974) y Polonia (Starmach, 1977) es más frecuente la poliecia.

La aparición de las rodofíceas de agua dulce en climas templados suele producirse en marzo-abril (Sheath \& Burkholder, 1983) mientras que la época de mayor crecimiento, producción de biomasa y frecuencia reproductora empieza a finales del otoño y se extiende hasta principios del verano (Israelson, 1942). Concretamente, $B$. boryanum puede encontrarse en fase reproductora en Suecia de marzo a septiembre (Kylin, 1912) y en Florida (USA) de septiembre a junio (Sheath $\&$ Burkholder, 1983), mientras que el desarrollo de $B$. moniliforme es ligeramente mas tardío: abril a octubre (Kylin, 1912) y octubre a agosto (Sheath \& Burkholder, 1983). En la zona de estudio es posible recolectar plantas fructificadas a lo largo de todo el año, tanto de

\begin{tabular}{|c|c|c|c|c|c|c|c|c|c|c|c|c|c|}
\hline & & $\mathrm{pH}$ & $\begin{array}{l}\mathrm{Ca}^{++} \\
\mathrm{mg} / \mathrm{l}\end{array}$ & $\begin{array}{l}\mathrm{Mg}^{++} \\
\mathrm{mg} / \mathrm{l}\end{array}$ & $\begin{array}{l}\mathrm{Na}^{+} \\
\mathrm{mg} / \mathrm{l}\end{array}$ & $\begin{array}{c}\mathrm{K}^{+} \\
\mathrm{mg} / \mathrm{l}\end{array}$ & $\begin{array}{l}\text { Dureza } \\
{ }^{\circ} \mathrm{F}\end{array}$ & $\begin{array}{c}\text { Conductiv } \\
\mu \mathrm{S} / \mathrm{cm}\end{array}$ & v. ${ }^{T^{a}}$ & $\underset{\mathrm{mg} / \mathrm{I}^{-}}{\mathrm{HCO}^{-}}$ & $\begin{array}{l}\mathrm{SO}_{4}= \\
\mathrm{mg} / 1\end{array}$ & $\underset{\mathrm{mg} / \mathrm{l}}{\mathrm{Cl}^{-}}$ & $\begin{array}{l}\mathrm{NO}_{3}^{-} \\
\mathrm{mg} / \mathrm{l}\end{array}$ \\
\hline & $\max :$ & 7 & 84 & 3 & 7 & 2,0 & 32 & 72 & 18,3 & 3 & 117 & 16 & 7 \\
\hline & $\min :$ & 6,9 & & & 5 & & 18 & & & & 23 & 13 & 3 \\
\hline & $\mathrm{x}$ : & 7,24 & 73,33 & 32 & 5,67 & 1,33 & 26,67 & 642 & 16,67 & 297,67 & 72 & 14,67 & 4,6 \\
\hline$n=$ & $\sigma:$ & 0,41 & 9,24 & 8,89 & 1,16 & 0,58 & 7,57 & 68,79 & 1,52 & 73,92 & 47,92 & 1,53 & 2, \\
\hline & & & & 32 & 2. & & & & & & & & 50 \\
\hline & $\min :$ & 6,99 & 12 & 6 & 3 & 0 & 13 & 5 & 1 & 21 & 19 & 1 & 3 \\
\hline moni & $\mathrm{x}$ : & 7,44 & 65,80 & 19,50 & 9,10 & 1,58 & 24,30 & 646,20 & 14,98 & 285,70 & 112,80 & 19,30 & 12,80 \\
\hline$n=1$ & $\sigma:$ & 0,28 & 26,17 & 10,18 & 6,08 & 0,83 & 7,03 & 147,32 & 2,75 & 47,34 & 98,86 & 6,60 & 16,97 \\
\hline
\end{tabular}

Tabla 2. Medias aritméticas, máximos, mínimos y desviaciones típicas de los principales parámetros físico-químicos del agua para B. boryanum y B. moniliforme. (Guara, M. \& Morell, I.; Inédito). Mean, maximum, minimum, and standard desviation of main physico-chemical parameters of water for $B$. boryanum and B. moniliforme. (Guara, M. \& Morell, I.; Inedit). 

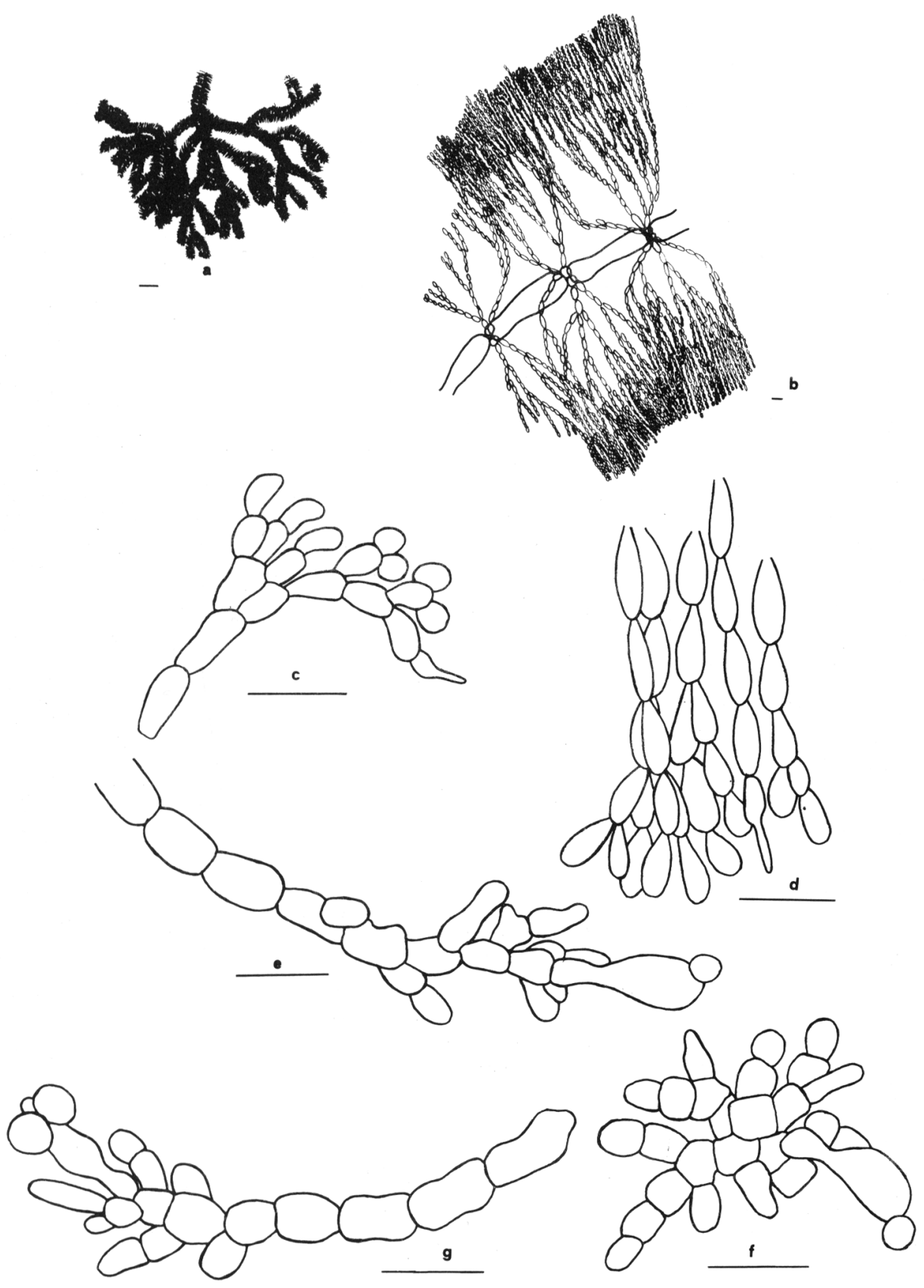

Figura 3. a: Aspecto general de B. boryanum (la escala representa $1 \mathrm{~cm}$ ); b: detalle de los verticilos; $\mathbf{c}$ : curvatura apical; d: pelos; e-g: variabilidad de las tricóginas. (La escala representa $10 \mu \mathrm{m}$ ). a: Habit of $B$. boryanum (the scale represents $1 \mathrm{~cm}$ ); $b$ : detailed view of the verticilles; $c$ : apical curvature; $d$ : hairs; $e$ g: tricogine variability. (The scale represent $10 \mu \mathrm{m}$ ). 
$B$. boryanum como de B. moniliforme. Observaciones similares se habían hecho ya para surgencias de la provincia de Alicante (Aboal, en prensa).

La amplitud altitudinal para $B$. moniliforme (290-1240 m) es mucho mayor que para $B$. boryanum $(300-520 \mathrm{~m})$. Parece probado, además, que $B$. boryanum prefiere zonas más bajas (Israelson, 1942) y que $B$. moniliforme es probablemente la especie que puede hallarse a mayor altitud (Johansson, 1982; Israelson, 1942).

En nuestros datos parece ligeramente más termófilo $B$. boryanum en consonancia con la menor altitud. Aunque la temperatura no parece ser un factor determinante de la distribución de estas especies (Sheath, 1984; Sheath \& Hambroock, 1990). Lo mismo puede decirse del $\mathrm{pH}$; en este caso ambos habitan aguas próximas a la neutralidad, pero su rango ecológico para este parámetro es más amplio para B. moniliforme (Johansson, 1982).

En la tabla II se compilan las principales características del agua para ambas especies. $B$. moniliforme crece en fuentes con mayor carga de nitratos, sulfatos y cloruros, en condiciones muy similares a las indicadas por Aboal (1989) y Sabater et al. (1989), mientras que $B$. boryanum prefiere aguas con mayor cantidad de cationes, fundamentalmente magnesio y calcio.

\section{BIBLIOGRAFÍA}

ABOAL, M. -1989- Aportación al conocimiento de las algas epicontinentales del SE de España. II. Rodofíceas (Rhodophyceae). Lazaroa, 11:115122.

ABOAL, M. (en prensa). Flora algal del Río Vinalopó.; Fundación Gil-Albert. Diputación Provincial de Alicante.

ALVAREZ COBELAS, M. -1984- Catálogo de las algas continentales españolas, II. Craspedophyceae; Cryptophyceae; Chrysophyceae; Dinophyceae; Euglenophyceae; Haptophyceae; Phaeophyceae; Rhodophyceae; Xantophyceae. Acta Bot. Malacitana, 9: 27-40

BOURRELLY, P. -1970- Les algues bleues et rouges. Les eugléniens, peridiniens et cryptomonadines. Boubée. Paris. 511 pp.

CAMBRA, J. -1985- Catalègs de les especies de cianofícies, d'algues continentals i del plàncton marí dels Països Catalans. Annex I: 501- 522. En: Historia Natural dels Països Catalans. Plantes inferiors. Vol. 4. Llimona, X. et al. Enciclopedia Catalana. Barcelona. $588 \mathrm{pp}$.

GIBSON, M.T. \& B.A. WHITTON -1987- Hairs, phosphatase activity and environmental chemistry in Stigeoclonium, Chaetophora y Draparnaldia (Chaetophorales). Br. Phycol. J., 22: $11-22$.

I.G.M.E. -1985- Mapa geológico de España. Vinarós 1:200.000. Servicio de Publicaciones Ministerio de Industria y Energía.

ISRAELSON, G. -1942- The freshwater florideae of Sweden. Studies on their taxonomy, ecology and distribution. Symb. Bot. Ups., 6 (1): 1- 135.

JOHANSSON, C. - 1982- Attached algal vegetation in running waters of Jämtland. Sweden. Acta Phytogeogr. Suec., 71: 1-84.

KYLIN, H. -1912- Studien über die schwedischen Arten der Gattungen Batrachospermum Roth und Sirodotia nov. gen. N. Acta Reg. Soc. Sci. Upsal. Ser. 4, Vol 4 (3): 1-40.

POVOA DOS REIS, M. -1974- Chaves para a identificaçao das especies portuguesas de Batrachospermum Roth. Ann. Soc. Brot., 40: $37-125$.

SABATER, S., M. ABOAL y J. CAMBRA -1989Nuevas aportaciones de rodofíceas en aguas epicontinentales del NE y SE de España. Limnetica, 5: 93-100.

SHEATH, R.G. -1984- The biology of freshwater red algae. Prog. Phycol. Res., 3: 89-157.

SHEATH, R.G. \& J.M. BURKHOLDER, -1983Morphometry of Batrachospermum populations intermediate between B. boryanum and B. ectocarpum (Rhodophyta) J. Phycol., 9: 324 331

SHEATH, R.G. \& J.A. HAMBROOCK -1990Freshwater ecology. In: Biology of the Red Algae. Cole, K. M. \& Sheath, R. G. Cap. 16. Cambridge University Press. Cambridge. 423453.

STARMACH, K. -1977-Phaeophyta-Brunatnice and Rhodophyta- Krasnorosty. Flora Slodkowodna Polski. Tomo 14. Polska Academia Navk.Warszawa. 425 pp.

Aceptado para su publicación en Julio de 1993

Dirección de las autoras. Dpto de Biología Vegetal. (Botánica). Facultad de Biología. Universidad de Murcia. Campus de Espinardo. 30100 Murcia. España. 